\title{
Cilostazol induced migraine does not respond to sumatriptan in a double blind trial
}

\author{
Katrine Falkenberg, Bára Óladóttir á Dunga, Song Guo, Messoud Ashina and Jes Olesen
}

\begin{abstract}
Background: Cilostazol is an inhibitor of phosphodiesterase 3 and thus causes accumulation of cAMP. It induces migraine-like attacks in migraine patients. Whether the cilostazol model responds to sumatriptan in migraine patients and therefore is valid for testing of future anti-migraine medications has never been investigated.

Methods: In a cross-over study, 30 patients received cilostazol (200 mg p.o.) on two separate days each day followed by oral self-administered placebo or sumatriptan $50 \mathrm{mg}$. We recorded headache characteristics and associated symptoms using a questionnaire. The 30 participants were asked to subsequently treat their spontaneous attacks with sumatriptan $(50 \mathrm{mg}$ ) or placebo in a double-blind cross-over design and 15 participants did so.
\end{abstract}

Results: Cilostazol induced headache with some migraine characteristics in all participants; 18 patients on the sumatriptan day and 19 patients on the placebo day fulfilled criteria for a migraine-like attack. The difference in median headache intensity between sumatriptan and placebo at $2 \mathrm{~h}$ was not significant $(p=0.09)$, but it was at $4 \mathrm{~h}(p=0.017)$. During spontaneous attacks, the difference between placebo and sumatriptan was not significant at $2 \mathrm{~h}(p=0.26)$, but it was highly significant at $4 \mathrm{~h}(p=0.006)$.

Conclusion: The cilostazol model in migraine patients could not be validated by a sufficient sumatriptan response. The model may perhaps respond to new drugs that act intracellularly or directly on ion channels.

Trial registration: The study is registered on clinicaltrials.gov (NCT02486276)

Keywords: Headache, Migraine, Pain, Phosphodiesterase type 3, Human migraine model

\section{Background}

The current treatments for migraine are not satisfactory and there remains a great need for new acute and prophylactic anti-migraine drugs. Testing new drugs in spontaneous migraine is cumbersome and it is done outside of the hospital which makes additional study of pharmacokinetic and -dynamics difficult. A valid experimental model could test new drugs under standardized circumstances and in a short period.

Since healthy volunteers are easiest to recruit and tablets are the preferred mode of administration of migraine treatment, we first tried to develop and validate

\footnotetext{
* Correspondence: Jes.olesen@regionh.dk

Danish Headache Centre and Department of Neurology, University of Copenhagen, Rigshospitalet Glostrup, Copenhagen, DK-2600, Glostrup, Denmark
}

a model in healthy subjects using isosorbide-5-mono nitrate (5-ISMN) [1] and cilostazol [2] as the headache inducing substances. In both trials, we used sumatriptan tablets to validate the model. Sumatriptan tablets had no effect on 5-ISMN induced headache [1], and only a trend toward efficacy on cilostazol induced headache in healthy volunteers [2]. Consequently, we now try to develop a model in patients with migraine using cilostazol as the headache inducing substance and sumatriptan to validate the model. Previous studies have shown that cilostazol causes migraine-like attacks in $86 \%$ of patients without aura [3, 4]. Cilostazol is an inhibitor of phosphodiesterase 3 (PDE3) which breaks down cyclic adenylate monophosphate (cAMP). When break down is inhibited, cAMP accumulates leading to general vasodilatation. Since cAMP accumulation is the only effect of cilostazol, 
cAMP must be the cause of headache/migraine-like attacks after cilostazol administration [3]. Whether the headache is a consequence of the vasodilatation or due to other effects of cAMP is not known. Whether the cilostazol model in migraine patients can be used to test current and future anti-migraine medications has never been validated. In a previous study we induced migraine-like attacks in $\mathrm{MO}$ patients with cilostazol and the patients treated the induced migraine attacks with a triptan in an open and uncontrolled fashion [3]. Patients responded well but at a late time after the provocation and perhaps due to the placebo effect. It is therefore necessary to test the efficacy of sumatriptan against cilostazol induced headache/migraine in a double-blind, cross-over study.

\section{Methods}

The method has previously been described in two studies on healthy volunteers by the authors $[1,2]$.

\section{Participants}

Thirty patients with migraine without aura were included. 23 of the patients were self-reported triptan responders and 7 were triptan-naive. Inclusion criteria were: Patients fulfilling IHS criteria for migraine without aura of both sexes, aged 18-60 years and weighing $45-95 \mathrm{~kg}$. Females were requested to use effective contraception.

Exclusion criteria were: Patients fulfilling any other type of headache than MO (except episodic tension-type headache $<1$ day per week), self-reported triptan nonresponders, serious somatic or psychiatric disease, pregnancy, and intake of daily medication (except oral contraceptives).

One participant dropped out for personal reasons. She was replaced with a new participant.

After completion of the study and with the knowledge of a partly negative response of sumatriptan to cilostazol headache, we wanted to be sure that the response was not due to lack of efficacy of sumatriptan in the study population. Therefore, we contacted all 30 participants and invited them to treat their spontaneous attacks with sumatriptan in a double-blind fashion. 2 patients did not suffer from migraine anymore, 2 patients were pregnant, 2 patients never responded to our request and 9 patients declined to participate. Fifteen patients accepted to treat their spontaneous attacks, and this post ad-hoc analysis was thus slightly underpowered.

\section{Design}

We conducted a double-blinded, randomized, balanced, placebo-controlled, cross-over study in which cilostazol $200 \mathrm{mg}$ was given orally on two separate days, five days or more apart. The provocation was both days followed by oral self- administrated placebo or sumatriptan $50 \mathrm{mg}$. The 15 patients, who subsequently treated their spontaneous attacks in a double-blind fashion, did so at least one month after the provocation study.

The central pharmacy of the Capital Region of Copenhagen performed the randomization of the experimental drug in a balanced fashion. The randomization code did not leave the hospital during the study and was not available to the investigators until after termination of the study. We did not break the code until data management took place.

\section{Standard protocol approvals}

All participants gave written, informed consent to participate in the study. The study was approved by the Ethics Committee of Copenhagen (H-8-2014-009), the Danish Data Protection Agency, and the Danish Medicines Agency. The study is registered on clinicaltrials.gov (NCT02486276) and was conducted according to the Helsinki II declaration of 1964, as revised in 2008.

All participants were enrolled via the website forsøgsperson.dk [5], through the Danish Headache Center or through patient organizations.

\section{Study procedure}

The patients had to be headache free $48 \mathrm{~h}$ prior to the study and not to have taken any type of painkillers $12 \mathrm{~h}$ before beginning of the study. A pregnancy test was taken at the beginning of each study day on all fertile female participants. All participants had two separate study days at least five days apart. They arrived nonfasting at the clinic between 8:00 a.m. and 12:00 a.m. Full medical history, physical examination, electrocardiography (ECG), vital signs and baseline headache were collected at arrival. All participants received cilostazol $200 \mathrm{mg}$ orally on both study days and went home immediately after. When the participants reached headache intensity 4 on the numerical rating scale (NRS) or six hours after cilostazol, the treatment (placebo or sumatriptan) was taken. All patients were thoroughly instructed about time of medication intake and noted time of intake in a questionnaire so the authors could ensure that treatment was taken appropriately. Time of treatment was chosen as a trade-off between treating early enough (sumatriptan is most effective when taking early in an attack) and not treating before migraine mechanisms were activated. Also, we needed the patients to have a measurable degree of headache before treatment. In case of severe headache not responding to the experimental treatment, the participants were allowed rescue with their usual anti-migraine medication or nonsteroidal anti-inflammatory drugs (NSAIDs) but not before two hours after placebo or sumatriptan. According to the patients' report, no one took rescue 
medication before $2 \mathrm{~h}$ after treatment (see Table 2) and we have to believe on the patients' report.

During the study, an emergency phone was always open where patients could call if they experienced severe headaches or discomfort.

The 15 patients, who treated their spontaneous attacks also took the treatment (sumatriptan or placebo) when headache intensity was 4 on NRS. The participants were also allowed rescue with their usual anti-migraine treatment two hours after the experimental drug, if the headache did not respond to the experimental drug.

\section{Headache parameters}

Headache parameters and accompanying symptoms were recorded by the investigator at baseline on a headache questionnaire. Afterward headache intensity, characteristics (unilateral/bilateral, quality and aggravation by physical activity), accompanying symptoms (nausea/ vomiting, phono- and photophobia) and side effects were scored on a self-administered questionnaire. The patients had to fill out the questionnaire every $30 \mathrm{~min}$ the first six hours after cilostazol and thereafter every hour until $12 \mathrm{~h}$ after cilostazol. The intensity was scored on a Numerical Rating Scale (NRS) from 0 to 10, 1 representing a very mild headache (including feeling of pressing or pulsation), 5 a headache of medium severity and 10 the worst possible headache (10). All patients had a thorough instruction for the questionnaire. The rational for a self-administered questionnaire is feasibility of the study. Few patients would accept staying at the hospital for over $12 \mathrm{~h}$ and especially not while having a migraine attack. So for the sake of the patients, they were allowed to treat at home.

One patient went to bed at $11 \mathrm{~h}$. To make it possible to plot the $0-12 \mathrm{~h}$ curve, the missing data at $12 \mathrm{~h}$ were filled in using last observation carried forward.

When the patients treated their spontaneous migraine attacks, they were instructed to fill out the questionnaire when they felt an attack and to keep doing so for at least $6 \mathrm{~h}$ after the experimental drug.

The following criterion was used for a migraine-like attack induced $0-12 \mathrm{~h}$ after administration of cilostazol:

Headache fulfilling criteria C and D for migraine without aura according to the IHS criteria [6].

C. C. Headache has at least two of the following characteristics:

- Unilateral location

- Pulsating quality

- Moderate or severe pain intensity (moderate to severe pain intensity is considered $\geq 4$ on NRS)

- Aggravation by cough or causing avoidance of routine physical activity
D. D. During headache at least one of the following:

- Nausea and/or vomiting

- Photophobia and phonophobia.

\section{Statistical analysis}

Calculation of sample size was based on the detection of a difference in headache intensity between two experimental days, at $5 \%$ significance with $90 \%$ power. We estimated that placebo had an effect on $20 \%$ and sumatriptan had $60 \%$ effect. Standard deviation was estimated based on previous data. The correlation between the two days was estimated conservatively at 0.5 . We also assumed no carry-over effect. We calculated that at least 18 participants should complete both experimental days. Due to uncertainty regarding these assumptions we decided to include 30 participants. The area under the curve (AUC) for headache score was used as a summary measure for analyzing differences between the groups and was calculated according to the trapezium rule (12). Pain-freedom is the recommended primary outcome parameter in clinical trials, but in experimental studies it is more powerful to score pain on Numerical Rating Scale (NRS) $0-10$ and use median headache score as outcome parameter. Even though the effect of triptans is higher at $4 \mathrm{~h}$ than at $2 \mathrm{~h}$, clinical migraine trials always study the effect of pain relieve after $2 \mathrm{~h}$. Therefore, our primary endpoints were (1) difference in median headache intensity between sumatriptan and placebo at two hours after treatment, and (2) difference in AUC 0-4 h between the two experimental days. Secondary endpoints were difference in pain intensity difference (PID) between the sumatriptan day and the placebo day, (PID is the difference in pain intensity at the various time points versus baseline. This will take headache score at treatment time into account (headache score varies from 0 to 6 with a median headache score at 4)), difference in median peak headache score, difference in median headache intensity between the two treatments at $4 \mathrm{~h}$, AUC 0-2 $\mathrm{h}$ after sumatriptan/placebo and accompanying symptoms as nausea, photo- and photophobia.

Headache intensity scores are presented as medians (range). Differences in median headache scores and AUC for headache scores were tested using the Wilcoxon signed rank test. Difference in pain intensity difference between the sumatriptan day and the placebo day were tested using Mann-Whitney $U$ test. The incidence of headache and associated symptoms were analyzed as binary categorical data with McNemar's test. Age and weigh are presented as means. We did not correct for multiple testing. All analyses were performed with SPSS for Windows 11.5 (Chicago, IL, USA), or GraphPad Prism version 7.0. A $p<0.05$ was considered significant. 


\section{Results}

Thirty patients with migraine without aura (22F, $8 \mathrm{M})$ completed both days of the provocation study. Mean age was 34.4 years (range 21-59 years) and mean weight was $69.8 \mathrm{~kg}$ (range 47-95 kg). Mean attack frequency per month was 3.9 (range 1-10). Fifteen of the 30 patients reported a first degree relative with migraine. All 30 patients were asked, but only 15 patients subsequently treated their spontaneous migraine attacks with sumatriptan and placebo in a double-blind cross-over fashion (Fig. 1).

\section{Cilostazol induced headache}

Cilostazol induced headache in all patients on both days (NRS range 1-10). The headache had several migrainelike features (summarized in Table 1); 23 patients (77\%) fulfilled criteria for a migraine-like attack on either one or both provocation days, 18 patients $(60 \%)$ on the sumatriptan day and 19 (63\%) on the placebo day. Median time to migraine attack was $4 \mathrm{~h}$ (range 2-8 $\mathrm{h}$ )

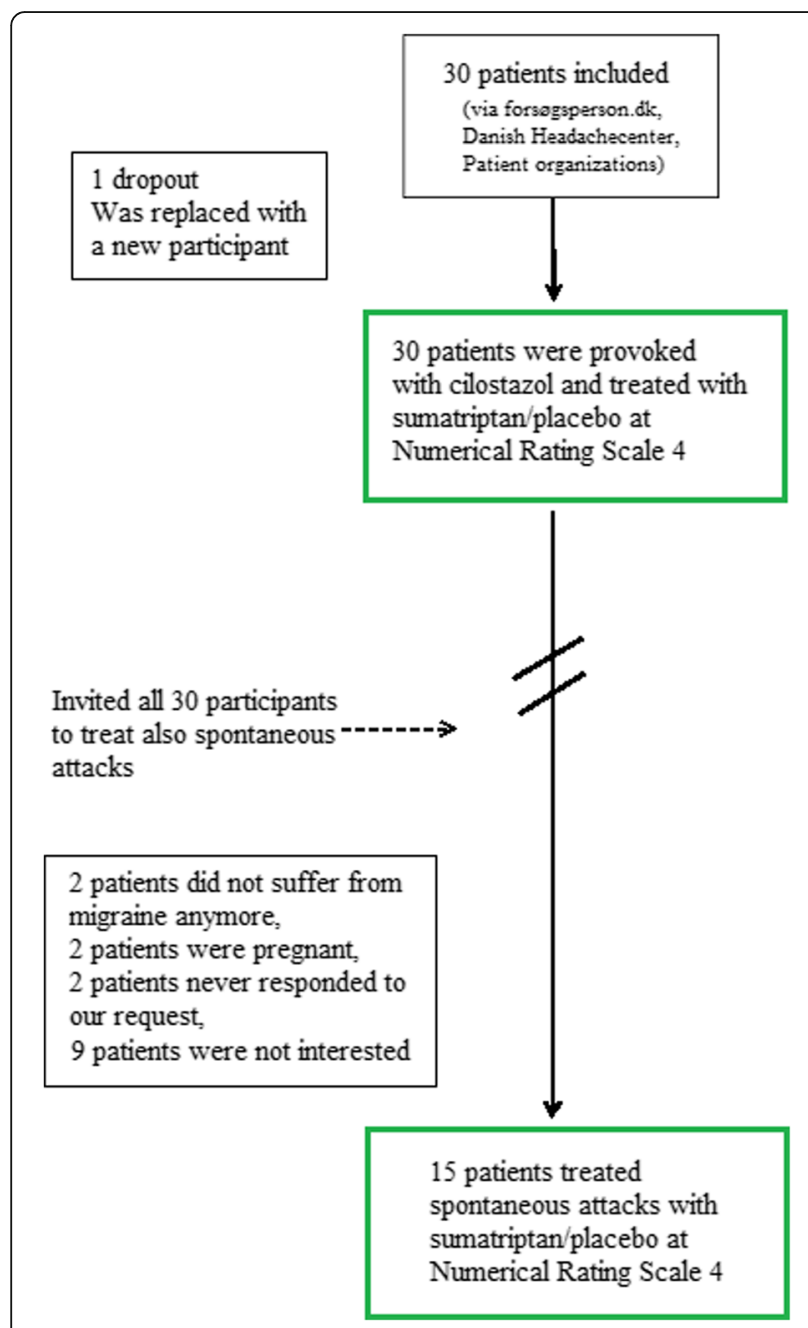

Fig. 1 Study enrolment on the sumatriptan day and $5 \mathrm{~h}$ (range $0.5-9 \mathrm{~h}$ ) on the placebo day. Headache characteristics and associated symptoms on the two experimental days are presented in Table 1. Characteristics of the headache for each subject are presented in Table 2. Median headache score $0-12 \mathrm{~h}$ after cilostazol for the two treatment groups is illustrated in Fig. 2. Note that the median headache score is reproducible between the two days until time of treatment. The figure also illustrates that median headache score at treatment was 4 (range 0-6) on both days and median time to treatment was $4.5 \mathrm{~h}$ (range 2-6 h) on the sumatriptan day and 4.25 $\mathrm{h}$ (range 1.5-6 h) on the placebo day.

\section{Effect of sumatriptan on cilostazol induced headache}

The difference in median headache intensity between sumatriptan and placebo at $2 \mathrm{~h}$ after treatment (our primary end-point), was not statistically significant $(p=0.09)$. At $2 \mathrm{~h}$, median headache score on the sumatriptan day was 4 and median headache score on the placebo day was 6 (Fig. 3). The increase in headache intensity $0-2 \mathrm{~h}$ after placebo was significant $(p<0.001)$ while there was no increase after sumatriptan (Fig. 3). The difference between pain intensity difference (PID) $2 \mathrm{~h}$ after treatment was significant $(p=0.04)$.

Median headache score $4 \mathrm{~h}$ after sumatriptan was 3 and median headache score after placebo was $5(p=0.017)$ (Fig. 3). Headache increased $4 \mathrm{~h}$ after placebo $(p=0.008)$, but not after sumatriptan $(p=0.28)$ (Fig. 3). PID at $4 \mathrm{~h}$ after placebo was significantly larger than after sumatrip$\tan (p=0.0005)$.

Another primary endpoint, difference in the area under the headache score curve (AUC) $0-4 \mathrm{~h}$ after treatment was not significant $(p=0.10)$. Neither was our secondary endpoint, difference in AUC $0-2 \mathrm{~h}$ after treatment $(p=0.26)$. Explorative analysis showed that AUC $0-6 \mathrm{~h}$ after treatment was significant with a $p$-value of 0.049 .

Another secondary end-point, difference in median peak headache score is illustrated in Fig. 4. Median peak headache score was 5 (range 1-10) on the sumatriptan day and 7 (range $1-10)$ on the placebo day $(p=0.03)$ (Fig. 4).

Rescue medication was taken by 25 patients on the sumatriptan day and by 29 patients on the placebo day. Median time to intake was $7 \mathrm{~h}$ and $6.5 \mathrm{~h}$ respectively and we do therefore not believe that intake of rescue medication have influenced our data.

\section{Response of spontaneous attacks to sumatriptan}

To test the sensitivity of the study population to sumatriptan, we asked all 30 participants to treat their spontaneous attacks in a double-blind fashion with sumatriptan and placebo. Fifteen patients completed this post-hoc study. 
Table 1 Clinical characteristics (our secondary end-point) of headache and associated symptoms after cilostazol

\begin{tabular}{|c|c|c|c|}
\hline & Sumatriptan $(n=30)$ & Placebo $(n=30)$ & $p$-value $^{a}$ \\
\hline Number of participants reporting headache & 30 & 30 & 1.00 \\
\hline \multicolumn{4}{|l|}{ (range 1-10 on NRS) } \\
\hline Median peak headache score (range) & $5(1-10)$ & $7(1-10)$ & $0.03^{b}$ \\
\hline \multicolumn{4}{|l|}{ No. of participants with } \\
\hline Unilateral location & 15 & 16 & 1.00 \\
\hline Throbbing headache & 19 & 21 & 0.63 \\
\hline Aggravation by physical activity & 26 & 27 & 1.00 \\
\hline Nausea & $17\left(2^{\mathrm{c}}\right)$ & $17\left(3^{\mathrm{c}}\right)$ & 1.00 \\
\hline Photophobia & 14 & 17 & 0.25 \\
\hline Phonophobia & 10 & 14 & 0.29 \\
\hline Rescue medication & 25 & 29 & 0.13 \\
\hline Migraine-like attack & 18 & 19 & 1.00 \\
\hline
\end{tabular}

${ }^{a}$ McNemar's test

${ }^{b}$ Wilcoxon signed rank test

'Subject vomited

Our primary end-point, difference in median headache score $2 \mathrm{~h}$ after treatment was not significant $(p=0.26)$. At $2 \mathrm{~h}$, median headache score after sumatriptan was 3 and median headache score after placebo was $5.4 \mathrm{~h}$ after treatment the headache score was significantly reduced after sumatriptan $(p<=0.001)$, but not after placebo $(p=0.32)$ and the difference between median headache score $4 \mathrm{~h}$ after the two treatments (our secondary end-point) was highly significant with a $p$-value of 0.006. At $4 \mathrm{~h}$ after treatment, median headache score after sumatriptan was 0 and median headache score after placebo was 3 . The difference in median headache score between the two treatment days was also significant at $5 \mathrm{~h}$ $(p=0.03)$ and at $6 \mathrm{~h}$ after treatment $(p=0.04)$. See Fig. 5 for an overview.

\section{Explorative analyses}

Explorative analyses showed that our results did not differ between sexes, pre-disposed vs. non-predisposed individuals, between patients fulfilling criteria for a migraine-like attack and those who did not, or between patients who did and did not participate in the second study treating their spontaneous attacks.

\section{Discussion}

Cilostazol induced headache in all 30 patients on both provocation days and provoked a migraine-like attack in more than half of the patients. Thus, we confirm previous data showing that cilostazol is a very powerful headache inducing substance ideal for human experimental migraine models [2, 3, 7]. Unfortunately our study did not show a clear cut effect of sumatriptan on the cilostazol induced headache. Therefore this model is not suitable for the testing of novel drugs, with a mechanism of action similar to sumatriptan.

\section{Was the lack of effect of sumatriptan due to the study population?}

Only 15 of the original 30 participants treated their spontaneous attacks. Thus, this study was slightly underpowered. Seven of the original 30 participants were triptan naïve. Only 2 of the 7 treated their spontaneous attacks in a double blind fashion. These 2 patients responded well to sumatriptan. At $2 \mathrm{~h}$ after treatment, sumatriptan showed a trend towards efficacy and $4 \mathrm{~h}$ after treatment the effect was highly significant. We explain the missing effect at $2 \mathrm{~h}$ with the small sample size as it is well known that the effect of triptans is higher at $4 \mathrm{~h}$ than at $2 \mathrm{~h}$ [8]. The lack of sumatriptan effect on cilostazol headache is therefore unlikely to be due to insensitivity of the study population.

\section{Can we use the present model to test new acute migraine drugs?}

This study represents the third attempt to validate a pragmatic human migraine model by its response to sumatriptan. Ideally an experimental model should respond to all known specific anti-migraine drugs (triptans (5-HT1B/D agonists), CGRP receptor antagonists and 5 -HT1F agonists) in double-blind trials. It is unlikely however that such an ideal model would ever be found since mechanisms of migraine are probably heterogenic. Since triptans are most commonly used and seem to perform slightly better than CGRP antagonists and 5TH1F agonists, we have used sumatriptan as the validating drug for our model $[9,10]$. Unfortunately, sumatriptan did not reduce cilostazol induced headache/migraine-like 
Table 2 Characteristics of the headache for each subject

\begin{tabular}{|c|c|c|c|c|c|}
\hline Subject & Peak headache & Characteristics $^{\mathrm{a}}$ & Associated symptoms ${ }^{\mathrm{b}}$ & Migraine-like attack $^{\mathrm{d}}$ & Rescue treatment (hours) \\
\hline 1: Suma & $5(5.5 h)$ & $+/+/+$ & $-/+/+$ & Yes (5 h) & Bonyl (7 h) \\
\hline 1: Placebo & $5(5.5 h)$ & $+/+/+$ & $-/+/+$ & Yes (4 h) & Bonyl (6 h) \\
\hline 2: Suma & $7(7 \mathrm{~h})$ & $-/-/+$ & $-/-/-$ & No & Bonyl (9 h) \\
\hline 2: Placebo & $8(3 h)$ & $+/+/+$ & $+/+/+$ & Yes (2 h) & Bonyl (8 h) \\
\hline 3: Suma & $5(7)$ & $-/-/+$ & $-/-/-$ & No & Bonyl (9 h) \\
\hline 3: Placebo & $10(10 h)$ & $-/-/+$ & $+/+/+^{c}$ & Yes (8 h) & Bonyl $(7.5$ h) + Treo (12.5 h) \\
\hline 4: Suma & $4(5.5)$ & $-/+/+$ & $-/+/-$ & No & Bonyl (10 h) \\
\hline 4: Placebo & $8(5.5$ h) & $-/+/+$ & $+/+/+^{c}$ & Yes $(5.5$ h) & Bonyl (5.5 h) - Threw it up \\
\hline 5: Suma & $8(3.5 \mathrm{~h})$ & $-/-/+$ & $+/-/-^{c}$ & Yes (2.h) & Bonyl (4.5 h) + lbuprofen \& Para (6 h) \\
\hline 5: Placebo & $7(4.5$ h) & $-/-/+$ & $+/-/-^{c}$ & Yes (3.5 h) & Bonyl (5 h) \\
\hline 6: Suma & $4(4.5 \mathrm{~h})$ & $-/+/+$ & $+/+/+$ & Yes (3.5 h) & Bonyl (5.5 h) \\
\hline 6: Placebo & $7(4.5 \mathrm{~h})$ & $+/+/+$ & $+/+/+$ & Yes (3 h) & Bonyl (6 h) \\
\hline 7: Suma & $9(8 \mathrm{~h})$ & $+/+/+$ & $+/+/+$ & Yes (3 h) & Bonyl (8 h) \\
\hline 7: Placebo & $8(11$ h) & $+/+/+$ & $+/+/+$ & Yes (8 h) & Bonyl (12 h) \\
\hline 8: Suma & $5(4.5$ h) & $-/+/+$ & $-/-1-$ & No & Bonyl (6 h) \\
\hline 8: Placebo & $7(6 h)$ & $+/+/+$ & $-/-/-$ & No & Zolmitriptan (6 h) \\
\hline 9: Suma & $6(6 h)$ & $+/-/+$ & $+/+/-$ & Yes (6 h) & Bonyl (8 h) + Sumatriptan (9.5 h) \\
\hline 9: Placebo & $6(5 \mathrm{~h})$ & $-/+/+$ & $+/+/-$ & Yes (3.5 h) & Bonyl \& Sumatriptan (4.5 h) \\
\hline 10: Suma & $1(2 \mathrm{~h})$ & $-1-1-$ & $-1-1-$ & No & None \\
\hline 10: Placebo & $1(2 \mathrm{~h})$ & $-/-/-$ & $-/-/-$ & No & None \\
\hline 11: Suma & $5(6 h)$ & $+/+/+$ & $+/-/-$ & Yes (6 h) & Sumatriptan (8 h) \\
\hline 11: Placebo & $4(5.5 h)$ & $+/+/+$ & $-/-/-$ & No & Bonyl (7 h) + Sumatriptan (11.5 h) \\
\hline 12: Suma & $5(3.5 \mathrm{~h})$ & $+/+/+$ & $+/+/-$ & Yes $(2.5 \mathrm{~h})$ & Sumatriptan \& Paracetamol (5 h) \\
\hline 12: Placebo & $7(3.5 \mathrm{~h})$ & $+/+/+$ & $+/+/+$ & Yes $(0.5 \mathrm{~h})$ & Sumatriptan \& Paracetamol (4 h) + Bonyl (5.5 h) \\
\hline 13: Suma & $5(4 h)$ & $+/+/-$ & $+/+/+$ & Yes (4 h) & None \\
\hline 13: Placebo & $8(5 h)$ & $+/+/+$ & $+/+/-$ & Yes (1 h) & Relpax (5 h) \\
\hline 14: Suma & $4(4.5 \mathrm{~h})$ & $+/+/+$ & $+/+/-$ & Yes (4 h) & Bonyl (6 h) + Rizatriptan (7 h) + Treo (8 h) \\
\hline 14: Placebo & $8(7 \mathrm{~h})$ & $+/+/+$ & $+/+/-$ & Yes (5 h) & Bonyl (7 h) + Rizatriptan (7.5 h) + Treo (9 h) \\
\hline 15: Suma & $8(5.5$ h) & $+/+/+$ & $+/+/+$ & Yes (4.5 h) & Bonyl (6.5 h) \\
\hline 15: Placebo & $5(6 h)$ & $-/+/-$ & $+/+/-$ & Yes $(6$ h) & Bonyl (9 h) \\
\hline 16: Suma & $5(4.5 \mathrm{~h})$ & $+/+/+$ & $+/-/+$ & Yes (4 h) & Bonyl (10.5 h) \\
\hline 16: Placebo & $8(8 \mathrm{~h})$ & $-/+/+$ & $+/-/+$ & Yes (5 h) & Bonyl (7.5 h) + Sumatriptan (7.5 h) \\
\hline 17: Suma & $7(9$ h) & $-/-/+$ & $+/-/-$ & Yes $(8 \mathrm{~h})$ & Bonyl (2 h) + Sumatriptan (11.5 h) \\
\hline 17: Placebo & $8(7 \mathrm{~h})$ & $-/-/+$ & $-/-/-$ & No & Bonyl \& Sumatriptan (6 h) \\
\hline 18: Suma & $4(6 \mathrm{~h})$ & $-/+/-$ & $-/-/-$ & No & None \\
\hline 18: Placebo & 7 (5.5 h) & $-/+/+$ & $-/+/-$ & No & Treo (5.5 h) \\
\hline 19: Suma & $10(3 \mathrm{~h})$ & $-/-/+$ & $+/-/+$ & Yes $(2.5 \mathrm{~h})$ & Sumatriptan \& Bonyl \& Paracetamol (4 h) \\
\hline 19: Placebo & $6(5 \mathrm{~h})$ & $-/-/+$ & $-/-/+$ & No & Bonyl\&Para.\& Suma (5.5 h) + Suma\&Para. (7.5 h) \\
\hline 20: Suma & $5(8 \mathrm{~h})$ & $+/+/+$ & $+/+/+^{c}$ & Yes (5 h) & Bonyl \& Sumatriptan (7 h) + Sumatriptan (9 h) \\
\hline 20: Placebo & $6(9 \mathrm{~h})$ & $+/+/+$ & $+/+/+$ & Yes $(5.5$ h) & Bonyl \& Sumatriptan (7.5 h) + Sumatriptan (9 h) \\
\hline 21: Suma & $4(4.5 \mathrm{~h})$ & $+/-/-$ & $-/-/-$ & No & Sumatriptan (9 h) \\
\hline 21: Placebo & $4(5 h)$ & $+/-/-$ & $-/-/-$ & No & Sumatripan (8 h) \\
\hline 22: Suma & $6(10 \mathrm{~h})$ & $+/-/+$ & $-/+/-$ & No & None \\
\hline 22: Placebo & $8(9 h)$ & $+/+/+$ & $+/+/+$ & Yes (9 h) & Sumatriptan (7 h) \\
\hline
\end{tabular}


Table 2 Characteristics of the headache for each subject (Continued)

\begin{tabular}{|c|c|c|c|c|c|}
\hline Subject & Peak headache & Characteristics $^{a}$ & Associated symptoms $^{b}$ & Migraine-like attack $^{d}$ & Rescue treatment (hours) \\
\hline 23: Suma & $4(3.5 \mathrm{~h})$ & $+/+/+$ & $-/-1-$ & No & None \\
\hline 23: Placebo & $9(7 \mathrm{~h})$ & $+/+/+$ & $+/-/-$ & Yes (7 h) & Sumatriptan (6 h) \\
\hline 24: Suma & $3(6 h)$ & $-/-/+$ & $-/-/-$ & No & Paracetamol (9 h) \\
\hline 24: Placebo & $4(8 h)$ & $-/-/+$ & $-/-/-$ & No & Bonyl (8 h) \\
\hline 25: Suma & $5(3.5)$ & $+/+/+$ & $+/-/-$ & Yes (3.5 h) & Bonyl (5.5 h) + Zolmitriptan (6.5 h) \\
\hline 25: Placebo & $7(7$ h) & $+/+/+$ & $+/-/-$ & Yes (4 h) & Bonyl (6 h) \\
\hline 26: Suma & $8(5 h)$ & $-/+/+$ & $-/+/-$ & No & Bonyl \& Paracetamol (8 h) \\
\hline 26: Placebo & $8(6 h)$ & $+/+/+$ & $-/+/-$ & No & Bonyl (7.5 h) \\
\hline 27: Suma & $9(6 \mathrm{~h})$ & $-/+/+$ & $+/+/+$ & Yes $(2.5 \mathrm{~h})$ & Sumatriptan $(6 \mathrm{~h})+$ Excedrin $(7 \mathrm{~h})$ \\
\hline 27: Placebo & $7(4.5$ h) & $+/+/+$ & $-/+/+$ & Yes (4 h) & Excedrin $(6.5$ h) + Bonyl (7 h) \\
\hline 28: Suma & $7(5 \mathrm{~h})$ & $-/+/+$ & $+/+/+$ & Yes (2.5 h) & Bonyl \& Sumatriptan (5.5 h) + Sumatriptan (8.5 h) \\
\hline 28: Placebo & $8(7 \mathrm{~h})$ & $-/+/+$ & $+/+/+$ & Yes (6 h) & Bonyl \& Sumatriptan ( 6.5 h) \\
\hline 29: Suma & $5(9 h)$ & $+/+/+$ & $+/-/-$ & Yes (8 h) & Bonyl (8.5 h) \\
\hline 29: Placebo & $2(10 \mathrm{~h})$ & $-/-/+$ & $-/-/-$ & No & Bonyl (8 h) \\
\hline 30: Suma & $7(8 \mathrm{~h})$ & $-/-/+$ & $-/-/-$ & No & Bonyl (9 h) \\
\hline 30: Placebo & $8(8 h)$ & $-/-/+$ & $-/-/+$ & No & Dolol (9 h) \\
\hline
\end{tabular}

${ }^{a}$ Characteristics: Location/quality/aggravation

${ }^{\mathrm{b}}$ Associated symptoms: Nausea/photophobia/phonophobia ( ${ }^{\mathrm{c}}=$ vomited)

${ }^{\mathrm{d}}$ Fulfilled criteria for an experimental induced migraine-like attack (hours)

Para $=$ Paracetamol, Suma $=$ Sumatriptan

attacks in $\mathrm{MO}$ patients but only prevented the headache from developing in intensity. As discussed below, we suggest several explanations for the lack of response. Regardless of explanations, the model proposed in this study cannot be used to test drug candidates that act on the same level of the migraine cascade as sumatriptan. We have previously tested the effect of sumatriptan on cilostazol and isosorbide-5-mononitrate (5-ISMN) induced headache in healthy volunteers, and the results were similar $[1,2]$. In a previous study, open and uncontrolled

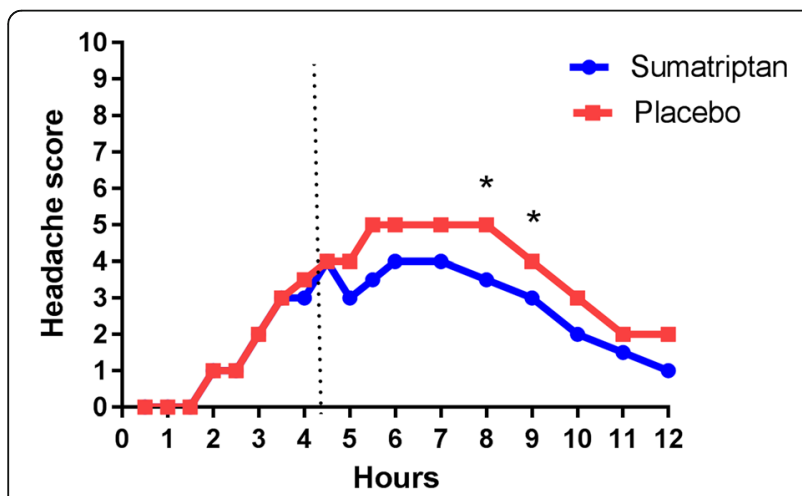

Fig. 2 Median headache score after cilostazol. Median headache score $0-12 \mathrm{~h}$ after cilostazol on the two treatment days. Median time to treatment was $4.5 \mathrm{~h}$ on the sumatriptan day and $4.25 \mathrm{~h}$ on the placebo day illustrated by the dotted line. The difference between the two treatment days is significant at $4 \mathrm{~h}(p=0.017)$ and $5 \mathrm{~h}$ $(p=0.028)$ after treatment

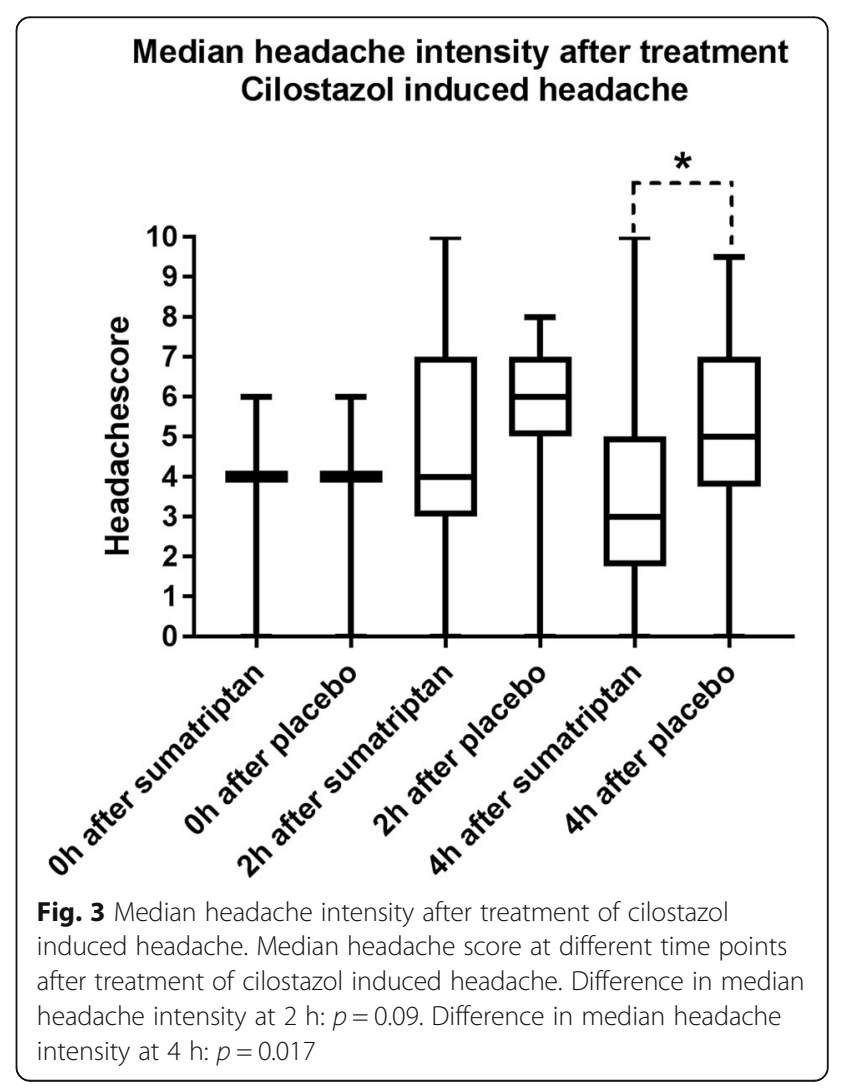




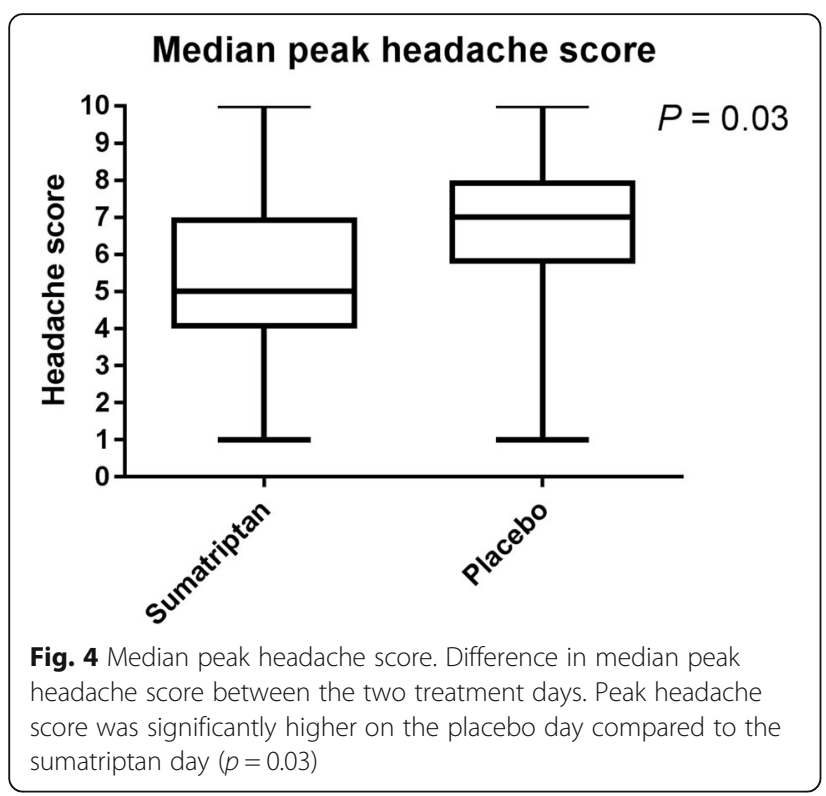

treatment with triptans of cilostazol induced migraine at a later time point showed an apparent positive result. The present study demonstrates the importance of the doubleblind design for the validation of drug responds.

\section{Mode of action: Sumatriptan and cilostazol in the migraine cascade}

Sumatriptan exerts its effect at several sites in the migraine cascade. It 1) Causes vasoconstriction via smooth muscle 5- $\mathrm{HT}_{1 \mathrm{~B}}$ receptors, 2) Hyperpolarizes afferent trigeminal fibers via $5-\mathrm{HT}_{1 \mathrm{D}}$ receptors, 3) Inhibits

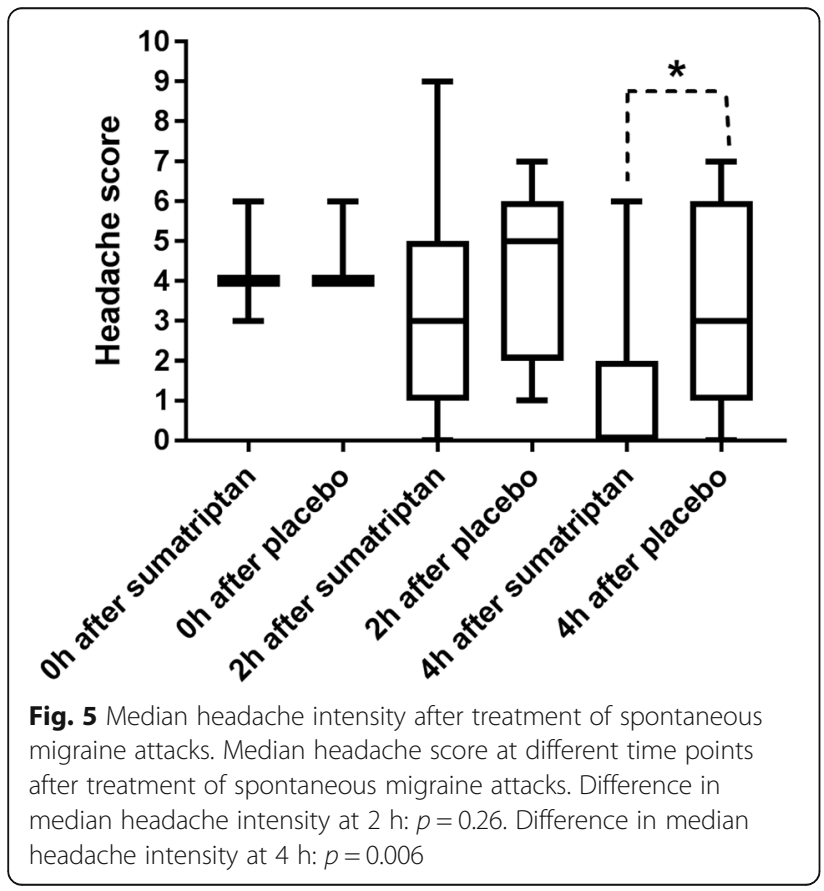

impulse transduction in the trigeminal nucleus via 5$\mathrm{HT}_{1 \mathrm{~B} / \mathrm{D} / \mathrm{F}}$ receptors, 4) Inhibits CGRP release [11-15]. All these actions happen via activation of $5-\mathrm{HT}_{1 \mathrm{~B} / \mathrm{D} / \mathrm{F}}$ receptors in the cell membrane. The receptors are coupled to G-proteins which inhibit adenylate cyclase and thereby decrease intracellular cyclic adenosine monophosphate (cAMP) [16-19] (see Fig. 6). Cilostazol is a selective inhibitor of Phosphodiesterase 3 (PDE3) which breaks down cAMP and hence it causes intracellular cAMP accumulation. PDE3 is located in the trigeminal ganglion, vascular smooth muscle cells and endothelium of cerebral and extra cerebral arteries [20,21].

The present study showed a trend towards efficacy of sumatriptan on cilostazol induced headache in migraine patients. The same trend was also present in healthy volunteers [2] and thus it seems likely that, although small, the effect is real. But sumatriptan cannot overpower the effect of cilostazol. We suggest three likely explanations for that: 1) Sumatriptan causes intracellular changes via an extracellular receptor on the cell membrane whereas cilostazol diffuses into the cell and acts directly on PDE3. In our previous study we used an NO-donor as the headache inducing substance, which also crosses the cell membrane and exerts its effect directly inside the cell [1]. Sumatriptan did not at all reduce that headache. Thus, it seems like the extracellular effect of sumatriptan cannot overpower compounds that intracellularly cause accumulation of second messengers (cAMP and cGMP) $[1,2] .2)$ Since there are several pathways in the body leading to increased cAMP, the production is only partly inhibited by sumatriptan and not enough to reverse the increase caused by cilostazol. 3) The half-life of cilostazol is $11 \mathrm{~h}$ and thus the migraine inducing effect of cilostazol continued long after the treatment with sumatriptan/placebo.

\section{The migraine inducing properties of cilostazol}

Eighteen $(60 \%)$ patients developed a migraine-like attack on the sumatriptan day and 19 (63\%) on the placebo day. Fourteen patients experienced a migraine-like attack on both provocation days and thus 9 patients did so on only one of the two days. Accordingly, our migraineinduction rate is less than the $86 \%$ previously reported $[3,4]$. There are several explanations why the induction in our study was less than previously. 1: We gave the patients treatment on both days. 2: Since our treatment was blinded we could not use the otherwise accepted criteria "Headache described as mimicking the patient's usual migraine attack and treated with acute migraine medication (rescue medication)". 3: Guo et al. showed that median time to migraine onset after cilostazol was $6 \mathrm{~h}$ [3], and Khan et al. [4] reported median $5 \mathrm{~h}$ until migraine attack. In our study median time to treatment was $4 \mathrm{~h}$ and the induction potential may very well be 


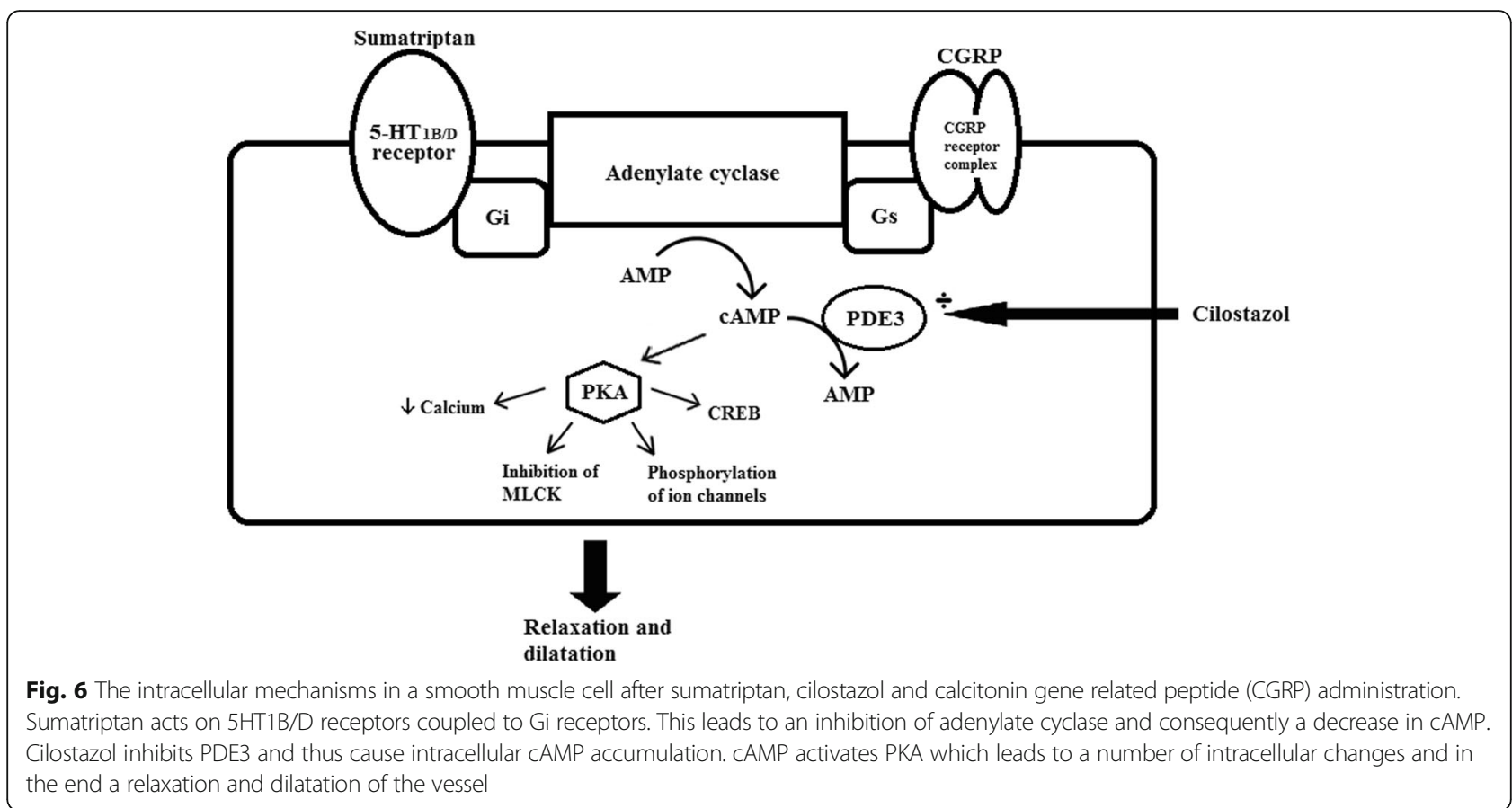

underestimated, as additional patients may have developed migraine if treatment had been postponed. However, the aim of the study was not to investigate the headache inducing potential of cilostazol and the lower induction rate will not change the outcome of our results.

\section{Strengths and weaknesses}

We calculated that 18 participants were needed and since we enrolled 30 participants, our study was well powered. Our study was placebo-controlled and, as discussed above, treatment efficacy can only be obtained by a double-blind study. Another strength is that we excluded triptan non-responders. A weakness was, however, that we included patients who had no experience with triptans. To compensate, we conducted a follow up study to test whether the spontaneous migraine attacks of the included patients responded to sumatriptan. The patients responded well and thus the lack of sumatriptan response in the provocation study could not be explained by insensitivity of the study population.

\section{Conclusion}

Cilostazol induced headache responds poorly to oral sumatriptan and hence may not be useful for testing novel drugs acting on membrane receptors. The model is, however, useful in studies of migraine mechanisms and may be useful in the testing of novel drugs acting deeper than sumatriptan in the migraine cascade (e.g. intracellularly or directly on ion channels activated by intracellular signaling).

\section{Additional file}

Additional file 1: Datasheet Cilostazol MO. (XLSX $25 \mathrm{~kb}$ )

\section{Abbreviations}

5-ISMN: Isosorbide-5-mononitrate; AUC: Area under the curve; CAMP: Cyclic adenylate monophosphate; CGMP: Cyclic guanosine monophosphate; CGRP: Calcitonin gene related peptide; ECG: Electrocardiography; NRS: Numerical rating scale; NSAIDs: Nonsteroidal anti-inflammatory drugs; PDE3: Phosphodiesterase 3; PID: Pain intensity difference

\section{Funding}

This work was supported by Candys Foundation, the Migraine Research Foundation, and the European Union's Seventh Framework program (2007-2013) under the grant agreement no. 602633 (FP7-Euroheadpain).

\section{Availability of data and materials}

The dataset supporting the conclusions of this article is included within the article's additional file.

\section{Authors' contributions}

$\mathrm{KF}, J \mathrm{O}, \mathrm{MA}$ and SG designed the experiment. KF and BD collected the data. KF analysed the data with guidance of SG. KF and JO wrote the manuscript. All authors read and approved the final manuscript.

Ethics approval and consent to participate The study was approved by the Ethics Committee of Copenhagen $(\mathrm{H}-8$ 2014-009). All participants received oral and written information and provided written informed consent before inclusion in the study.

\section{Consent for publication}

Not applicable

\section{Competing interests}

The authors declare that they have no competing interests

\section{Publisher's Note}

Springer Nature remains neutral with regard to jurisdictional claims in published maps and institutional affiliations. 
Received: 22 November 2017 Accepted: 24 January 2018

Published online: 02 February 2018

\section{References}

1. Hansen EK, Olesen J (2017) Towards a pragmatic human migraine model for drug testing: 2. Isosorbide-5-mononitrate in healthy individuals. Cephalalgia 37:11-19

2. Hansen EK, Guo S, Ashina M et al (2016) Toward a pragmatic migraine model for drug testing: I. Cilostazol in healthy volunteers. Cephalalgia 36: $172-178$

3. Guo S, Olesen J, Ashina M (2014) Phosphodiesterase 3 inhibitor cilostazol induces migraine-like attacks via cyclic AMP increase. Brain 137:2951-2959

4. Khan S, Deen M, Hougaard A et al (2017) Reproducibility of migraine-like attacks induced by phosphodiesterase-3-inhibitor cilostazol. Cephalalgia 033310241771975

5. National Center for clinical trial registrations. Raske forsøgspersoner søges til migræneforsøg. www.forsøgsperson.dk, (2017, Accessed 28 Aug 2017).

6. (IHS) HCC of the IHS (2013) The International Classification of Headache Disorders, 3rd edition (beta version). Cephalalgia 33:629-808

7. Birk S, Kruuse C, Petersen KA et al (2006) The headache-inducing effect of cilostazol in human volunteers. Cephalalgia 26:1304-1309

8. Evaluation of a multiple-dose regimen of oral sumatriptan for the acute treatment of migraine. The Oral Sumatriptan International Multiple-Dose Study Group. Eur Neurol 1991; 31: 306-313.

9. Ho TW, Mannix LK, Fan X et al (2008) Randomized controlled trial of an oral CGRP receptor antagonist, MK-0974, in acute treatment of migraine. Neurology 70:1304-1312

10. Hougaard A, Tfelt-Hansen P (2015) Review of dose-response curves for acute antimigraine drugs: triptans, 5-HT $1 F$ agonists and CGRP antagonists. Expert Opin Drug Metab Toxicol 11:1409-1418

11. Durham PL, Russo AF (2002) New insights into the molecular actions of serotonergic antimigraine drugs. Pharmacol Ther 94:77-92

12. Olesen J, Ashina M (2011) Emerging migraine treatments and drug targets. Trends Pharmacol Sci 32:352-359

13. Juhasz G, Zsombok T, Jakab B et al (2005) Sumatriptan causes parallel decrease in plasma calcitonin gene-related peptide (CGRP) concentration and migraine headache during nitroglycerin induced migraine attack. Cephalalgia 25:179-183

14. Eltorp CT, Jansen-Olesen I, Hansen AJ (2000) Release of calcitonin generelated peptide (CGRP) from guinea pig dura mater in vitro is inhibited by sumatriptan but unaffected by nitric oxide. Cephalalgia 20:838-844

15. Ahn S-K, Khalmuratova R, Jeon S-Y et al (2009) Colocalization of 5-HT1F receptor and calcitonin gene-related peptide in rat vestibular nuclei. Neurosci Lett 465:151-156

16. Barnes NM, Neumaier JF. Tocris Bioscience Scientific Review Series Neuronal 5-HT Receptors and SERT. http://www.komabiotech.co.kr/pdf/5-ht_ receptors_review.pdf (Accessed 27 March 2017)

17. Ferrari MD, Saxena PR (1995) 5-HT1 receptors in migraine pathophysiology and treatment. Eur J Neurol 2:5-21

18. Adham N, Kao HT, Schecter LE et al (1993) Cloning of another human serotonin receptor (5-HT1F): a fifth 5-HT1 receptor subtype coupled to the inhibition of adenylate cyclase. Proc Natl Acad Sci U S A 90:408-412

19. Miller KJ, King A, Demchyshyn L, Niznik H, Teitler M (1992) Agonist activity of sumatriptan and metergoline at the human 5-HTtD receptor: further evidence for a role of the 5-HT D receptor in the action of sumatriptan. Eur J Ptlarmacolo:99-112

20. Nordgaard JC, Kruse LS, Møller M et al (2013) Phosphodiesterases 3 and 5 express activity in the trigeminal ganglion and co-localize with calcitonin gene-related peptide. Cephalalgia 0:1-11

21. Degerman E, Belfrage $P$, Manganiello VC (1997) Structure, Localization, and Regulation of cGMP-inhibited Phosphodiesterase (PDE3). J Biol Chem 272: 6823-6826

\section{Submit your manuscript to a SpringerOpen ${ }^{\circ}$ journal and benefit from:}

- Convenient online submission

Rigorous peer review

- Open access: articles freely available online

- High visibility within the field

Retaining the copyright to your article

Submit your next manuscript at $\boldsymbol{\nabla}$ springeropen.com 